\title{
Analysis of acquired management competencies in education process
}

\author{
Alina Sherstneva* \\ Basic Training Department, Novosibirsk State University of Economics and Management, 630099 \\ Novosibirsk, Russia
}

\begin{abstract}
On the basis of practical experience, the author considers the existing system of learning future specialists in the management-related area for higher education level. The same acquired knowledge can be included in different competencies, but at the same time it changes significantly. Therefore, competence transforms the existing knowledge to adapt them to a given subject and to make it inseparably linked with professional actions. Combination of skills, knowledge and attitude and their interconnection form the internal structure of competence. The article considers a particular analysis of the new tasks facing the manager to model the competence that the manager lacks and to offer a special training program. The article is focused on professional competence development. The competence of a modern Russian manager presupposes the development and implementation a complex of modern professional knowledge and practical skills, their application, and the acquisition of effective social management skills and human studies technologies.
\end{abstract}

\section{Introduction}

The modern world is changeable and variable. The speed of change has increased significantly: what have taken years earlier now is being transformed in a few days. The old methods of management in terms of changed business climate are ineffective. Companies are forced to look for new methods of saving the competitiveness.

It should be noted, that the employees have also changed. The so-called representatives of generations $\mathrm{Y}$ and $\mathrm{Z}$ enter the labor market. They no longer have enough directives and orders, they want to participate in decision-making, develop and improve their skills. it is interesting to note that entrepreneurship has become a youth phenomenon as entrepreneurial intentions among young people have grown, whereas these have fallen among older people. That's why the role of manger becomes significant.

Talking about the role of a manager in transforming a group into an effective team, it should be mentioned that, first of all, managers use different motivation technologies. Forming a vision of the company's future, they always rely on the moral values essential for the team. This allows to add value to the work in the eyes of employees. Managers constantly involve team members in the discussion of the implementation of the projects. People feel their involvement in decision-making in the company, feel the importance of their opinion.

\footnotetext{
* Corresponding author: shers7neva@ gmail.com
} 
The manager strongly supports the efforts of employees to bring corporate values to life. Managers value and reward the personal achievements of their employees. Such a management approach allows to develop leadership skills of team members and the work becomes a source of inspiration.

Every company, employing a specialist, expects from him the high-quality performance. Active, task-oriented, talented people are always the key to success and the great advantage of the company. Doing a job, an individual does it in other people environment and automatically becomes a member of the group, in which he is carrying out working practice.

Entering the company, an individual analyses and evaluates the results of his job, analyses his coworkers experience, cooperating with chiefs, what allows to draw conclusions about the success of interaction with corporate culture. His findings lead to changing of his behavior in order to adapt in the company.

Outstanding teams do not appear as a result of the arrival of new people. Such teams are the result of developing a certain behavior. Effective teams use available components and turn them into the unique products, have broken individual efforts into common result. They discuss and coordinate important decisions, take responsibility for their actions.

The transformation of a working group into an effective team is impossible without a manager. It is the leader, who guides, develops, inspires and motivates the team; the task of the leader is to find contradictions and use them as a source of non-standard ideas.

A manger is a person who inspires people, inspires enthusiasm in employees through transmitting them his vision of the future; helping them to adapt to the new order, to pass the stage of changing.

\section{Problem statement}

When entrepreneurship is discussed and debated it is typically education for management that people have in mind. This approach is typically practice-oriented and aims to foster both business-oriented skills and enterprising skills. The focus is thus both on the content and the teaching methods. Still, it is education about entrepreneurship, that is, education focusing on transmitting declarative knowledge about what management is and what managers do, that is, by far, the most common educational approach. There is, however, a third approach to management education that is relatively different from the other two.

Education through entrepreneurship is an approach that focuses on using entrepreneurship as a teaching method. This approach to management education is closely related to the concept «enterprise education», as the goal is much broader compared to education for and about management. Whereas education for and about management typically focuses on new venture creation, the focus of education through entrepreneurship is more on fostering innovative, creative and enterprising individuals. The educational content in this approach does not need to focus on management, at least not in its contextual definition as an organizational form characterized by small business and new venture creation. The focus of this approach is rather on the pedagogy and the teaching methods. The lack of entrepreneurship-oriented content makes it questionable if this approach can really be regarded as education in entrepreneurship.

However, it should be emphasized that there is a clear focus on fostering management skills, such as creativity, resource marshalling and ambiguity tolerance, and similar to the other two approaches, it is by studying entrepreneurs, how they learn and act and how management motivation that this approach to education has developed [1-2]. 


\section{Materials and methods}

Due to tough competition and increased variability in the modern business world, the task of a manager is primarily to be able to worthily cope with changes. The more serious are changes, the greater the need for effective leadership.

Investigating the role of a manager in the management process, it's necessary to consider the main functions to perform. There are general and specific management functions. Specific functions are functions that include the management of any sphere, such as:

- production;

- economic;

- scientific and technological progress management.

General management functions are functions, that are performed by the manager, independently of the sphere of activity:

- organization;

- coordination and regulation;

- motivation.

Overall, the main difference is that traditional management allows to cope successfully with routine duties and entrepreneurship allows to implement the great plans, causing employees to feel involved in the common activity, the desire for achievements, self-esteem.

The ability of a manager to inspire enthusiasm in people, instill confidence in the correctness of the chosen course, encourage the team to cooperate and make a conscious choice of the way of changing, gives people confidence that they are able to overcome all obstacles.

The study was launched at Novosibirsk State University of Economics and Management in January 2021. Its main focus to develop a link between education process and work opportunities. For this reason the article contribute to comprehence and promotion of management competence in education system. The article ultimately aims to facilitate learning and ideas exchange among students and eventually have a positive impact for employability and active participation of students in society and the economy.

The survey aims to first of all, describe and get identification key competencies and components that every competence consist of. Secondly, on the given information to develop common structure by establishing a shared conceptual model that should be clear and transparent for all participants and lead to deeply understanding general components. And, as a result, to work out a number of learning outcomes in accordance with education framework about concept model that students have to know, can and use for a certain level of proficiency in aquired specialisation.

By establishing a common basis that initiatives dealing with the promotion of management as a competence across levels of education, purposes of application this concept can refer to unleash student's potential to participate in all areas of society by transforming ideas into action [3-5]. In survey it has used a robust mixed-method research process and each of its interim outputs has been validated through multi-stakeholder consultations, leading to progressive refinement and eventually to the consolidated framework presented in this article.

\section{Practical results}

In this part we will present the latest results from our longitudinal survey, which focuses on the effects of different approaches to management education on university students. The programs included in this study are all practice-based and have a strong focus on entrepreneurship; nevertheless, they have very different effects on students. We have sourced these differences in effects to the programs' focus on two different professional areas, both 
of which are closely connected with management, but in first case, we ask first and secondyears students who are without or just with basic experienced in their profession and second case master's student who are already involved in practical solutions during their work experience.

For sure bachelor's program have affected the students' entrepreneurial activities and entrepreneurial self-efficacy. We have further analyzed the extent to which the programs have changed their prior perception of their self-efficacy, and which types of student's prior contextual knowledge are particularly suitable for.

The survey was conducted among bachelor and master students of Novosibirsk State University of Economics and Management and include questions about roles of a manager and their understanding of future specialty.

The biggest part of bachelor's students is believed that manager is a person who coordinates someone, something, owns information about it and can provide it, negotiates within the framework of his responsibilities. In addition, personal characteristic was mentioned quite often. Manager is considered as a person who is sociable, friendly, charismatic, has many connections, understands well the mood of people and their needs for a product or service.

For sure, there is no clear understanding of acquired competencies during educational processes, more often some guess about future position. Common answer about a position as a mystery, often a human manager performs several tasks at once. Probably, this is such a local manager, not like a director who oversees the work of the enterprise as a whole, into a mini-manager who is responsible for his area.

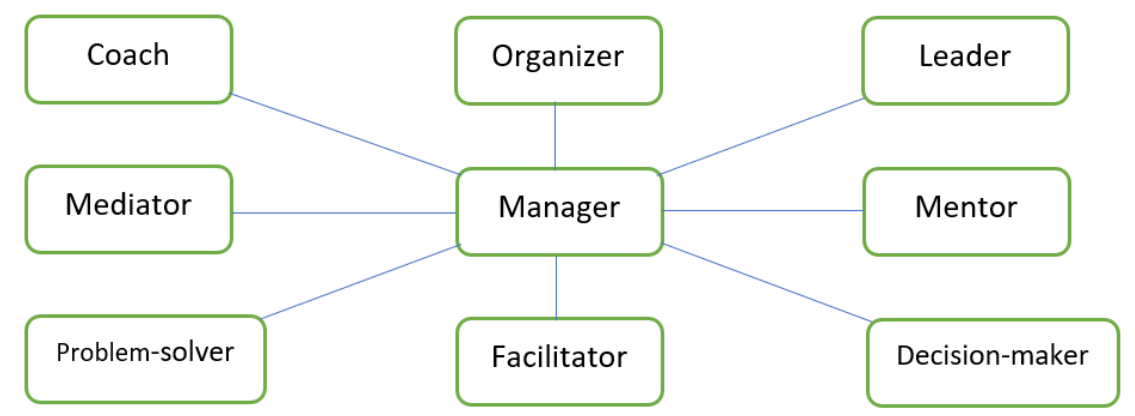

Fig. 1. Role of a manager.

Interviewing of master's student shows much more deeply understanding of manager's requirements. The focus on human resources plays a vital role in every organization, no matter how big or small it is, because the most valuable resource of any company is its people. Common profile of a modern manager is a strategic manager, whose responsibilities incorporates the formation of the company's staff policy, which includes the development of a personnel training system, maintaining a healthy psychological climate in the team, and motivating employees. The manager's main task is to determines the number of necessary staff, their development, and motivation.

This profession requires strong communication skills and practice in work with people, which is a feature of prime necessity. The most important roles and opportunities are presented below. 


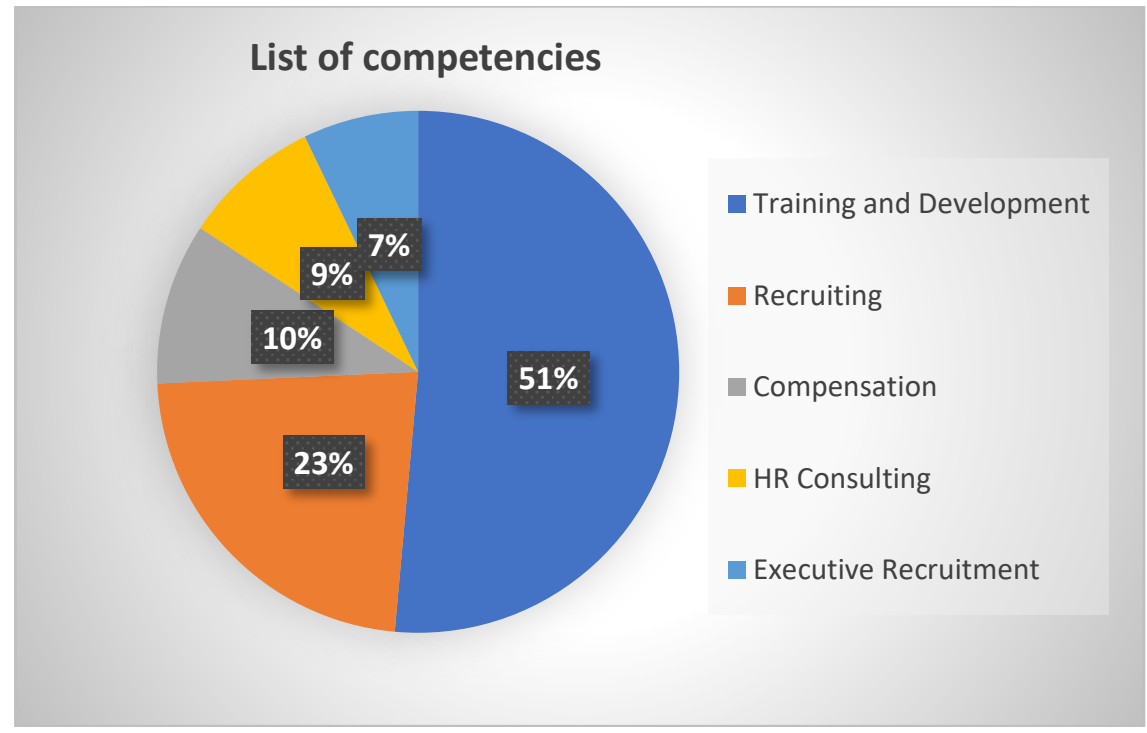

Fig. 2. List of competencies.

Specialists in training area should be aware of the latest trends in the development of personnel and actively use them in their own organization. It requires a thorough knowledge of company professional needs and ample experience in human resources training. The main goal is to keep the staff in the company, develop it to meet the challenges of the industry, and cultivate future leaders and managers among employees.

Recruiting is one of the most dynamic specializations in human resources. Recruiters actively seek out talented employees and attend recruitment events at schools and universities to attract new talent to their company. Recruiters present their company to candidates, being the first faces of a company that job seekers see, that is why they should have excellent communication and listening skills. This HR specialist is both a psychologist and an analyst who considers a person not only in terms of their professional competencies but also from the perspective of personal qualities, mindful that the human factor is important in teamwork. Recruiters are responsible for curating resumes of job seekers, and making recommendations to the human resources department for specific hires on the basis of experience and personal interaction.

Compensation and Benefits is focused on employee compensation and benefits policymaking. Experts in this field are well versed in the financial issues of the organization and work closely with the accounting department. These specialists must also be able to suggest appropriate compensation and benefits packages for each new hire and current employee on the basis of experience, seniority, and company contribution.

HR Consulting direction is gaining popularity as it is used by both small and large companies. Smaller companies cannot always afford the cost of a full HR department that is why they resort to outsourcing and hire HR consulting experts. Large corporations also use the services of consulting agencies. This is how they do routine audits of their own HR departments or mass recruitment of employees.

Executive Recruitment differs from standard recruiting because these professionals look for qualified executives to take on leadership roles within a company. While they still create job postings and interview candidates, they also use their own personal and professional contacts to learn about candidates not yet on the market. Executive recruitment managers are responsible for finding executives who can fill roles like Chief Financial Officer, Chief Executive Officer, and other department heads. 
According to interviewing students have already involved in working processes, this area is quite new but sufficient in every organization. As technology advances and the workforce changes, organizations need human resources to recruit, retain and engage their employees. Part of human resources is interesting from a professional standpoint as it demands to be a gregarious person who can identify the strong points in other people.

\section{Conclusion}

The analyses presented in this article indicate that ownership and the inclusion of the students' prior contextual knowledge in the educational process are important dimensions to focus on in educational programs for entrepreneurship. There are many positive outcomes for the programs on our sample, for all of the programs that have a strong focus on these dimensions. The students significantly increase their motivation levels in education after clear understanding of future roles. It is often necessary in these projects to comply with the professional values that are practiced by the partnering organizations, and the margin for errors is thus limited. This favors predictive methods over trial-and-error procedures, and the students often remain in their student role rather than adopting an entrepreneurial perspective. This does not mean that we should cease this type of education. However, our analyses show that it is important to consider how we can mimic entrepreneurial learning, include the students' prior contextual knowledge and increase the students' psychological ownership levels in these types of programs.

Students who already have a pre-knowledge and professional background are harder to increase the students' focus on management skills when they already have a high confidence in their ability to perform these skills, the comparison of the two groups becomes a bit unfair.

Another interesting finding in our analyses is that the students' perceived entrepreneurial social capital has a very positive influence. Exercises where students have to consider which types of contacts and resources, they have in their network could be one way of doing this. Another way could be to task the students with proactively extending their entrepreneurial network, by for example seeking out potential stake-holders, use professional networks to establish contacts, visit entrepreneurship associations and student incubators, and so forth.

We should, however, be careful when interpreting the result of the analyses since there are some limitations that need to be considered. The educational treatment is not randomly assigned to the students. This creates a self-selection bias in the sample and we can see that students in the instrumental programs have significantly higher initial values in the dimensions. There may well be positive effects of these programs that first reveal themselves long after the programs have ended.

The article was aimed to convince that management and management competencies are something that can be taught, and that the question is a case of how to best teach the subject at different levels of education and to different groups of students. But this conviction has often been challenged by people who believe that entrepreneurship has a biological explanation (inherited) rather than a cultural/ social one (environment) [6-10].

The study shows that self-employed parents' influence on their children's entrepreneurial attitudes are predominantly determined by culture, is therefore important because it shows that management can be taught.

That management can be explained through social factors is also under consideration supported by our analysis results. 


\section{References}

1. A. Sherstneva, Impact of modern processes on management education on the acquired competencies, New Industrialization and Digitalization, 93 (2020)

2. A. Sherstneva, Analyses of Aspects on an Entrepreneurial Mindset, Smart Innovation, Systems and Technologies, 139 (2019)

3. M. Bacigalupo, P. Kampylis, Y. Punie, G. Van den Brande, EntreComp: the entrepreneurship competence framework, Publication Office of the European Union, Luxembourg (2016)

4. A. Sherstneva, Algorithm development for the formation of entrepreneurial activity in educational process, Advanced in economics, business and management research, 81 (2019)

5. K. Moberg, H. Barslund Fosse, A. Hoffman, M. Junge, Impact of entrepreneurship education in Denmark, Danish foundation for entrepreneurship, 94 (2015)

6. R. Baron, Entrepreneurship: An evidence-based guide (Cheltenham, UK, Northhapton, MA, USA, 2012)

7. F. Cunha \& J. J. Heckman, Investing in Our Young People (Cambridge University Press, New York, 2010)

8. W.B. Gartner, N.M. Carter, Entrepreneurial behaviour and firm organizing processes (Boston, Kluwer, 2003)

9. J. Heinonen, U. Hytti, Entrepreneurship and Innovation, 11 (2010)

10. L Rosendahl Huber, R. Sloof, C.M. Van Praag, European Economic Review 72 (2014) 